\title{
Search for a Higgs boson decaying to weak boson pairs at LEP
}

\section{L3 Collaboration}

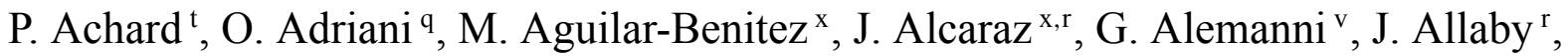
A. Aloisio $^{\text {ab }}$, M.G. Alviggi ${ }^{\text {ab }}$, H. Anderhub ${ }^{\text {at }}$, V.P. Andreev ${ }^{\text {f,ag }}$, F. Anselmo ${ }^{\text {h }}$, A. Arefiev ${ }^{\text {aa }}$, T. Azemoon ${ }^{\text {c }}$, T. Aziz ${ }^{\mathrm{i}, \mathrm{r}}$, P. Bagnaia ${ }^{\mathrm{al}}$, A. Bajo ${ }^{\mathrm{x}}$, G. Baksay ${ }^{\mathrm{y}}$, L. Baksay $^{\mathrm{y}}$, S.V. Baldew ${ }^{\text {b }}$, S. Banerjee ${ }^{\text {i }}$, Sw. Banerjee ${ }^{\mathrm{d}}$, A. Barczyk ${ }^{\text {at, ar }}$, R. Barillère ${ }^{\mathrm{r}}$, P. Bartalini ${ }^{\mathrm{v}}$, M. Basile ${ }^{\mathrm{h}}$, N. Batalova ${ }^{\mathrm{aq}}$, R. Battiston ${ }^{\text {af }}$, A. Bay ${ }^{\mathrm{v}}$, F. Becattini ${ }^{\mathrm{q}}, \mathrm{U}^{\mathrm{b}}$ Becker ${ }^{\mathrm{m}}$, F. Behner ${ }^{\text {at }}$, L. Bellucci ${ }^{\mathrm{q}}$, R. Berbeco ${ }^{\mathrm{c}}$, J. Berdugo ${ }^{\mathrm{x}}$, P. Berges ${ }^{\mathrm{m}}$, B. Bertucci ${ }^{\text {af }}$, B.L. Betev ${ }^{\text {at }}$, M. Biasini ${ }^{\text {af }}$, M. Biglietti ${ }^{\text {ab }}$, A. Biland ${ }^{\text {at }}$, J.J. Blaising ${ }^{\text {d }}$, S.C. Blyth ${ }^{\text {ah }}$, G.J. Bobbink ${ }^{\text {b }}$, A. Böhm ${ }^{\text {a }}$, L. Boldizsar ${ }^{1}$, B. Borgia ${ }^{\text {al }}$, S. Bottai ${ }^{\text {q }}$, D. Bourilkov ${ }^{\text {at }}$, M. Bourquin ${ }^{t}$, S. Braccini ${ }^{t}$, J.G. Branson ${ }^{\text {an }}$, F. Brochu ${ }^{\mathrm{d}}$, J.D. Burger ${ }^{\mathrm{m}}$, W.J. Burger ${ }^{\text {af }}$, X.D. Cai ${ }^{\mathrm{m}}$, M. Capell ${ }^{\mathrm{m}}$, G. Cara Romeo ${ }^{\text {h }}$, G. Carlino ${ }^{\text {ab }}$, A. Cartacci ${ }^{\mathrm{q}}$, J. Casaus ${ }^{\mathrm{x}}$, F. Cavallari ${ }^{\text {al }}$, N. Cavallo ${ }^{\text {ai }}$, C. Cecchi ${ }^{\text {af }}$, M. Cerrada ${ }^{\mathrm{x}}$, M. Chamizo ${ }^{\mathrm{t}}$, Y.H. Chang ${ }^{\text {av }}$, M. Chemarin ${ }^{\text {w }}$, A. Chen ${ }^{\text {av }}$, G. Chen ${ }^{\mathrm{g}}$, G.M. Chen ${ }^{\mathrm{g}}$, H.F. Chen ${ }^{\mathrm{u}}$, H.S. Chen ${ }^{\mathrm{g}}$, G. Chiefari ${ }^{\text {ab }}$, L. Cifarelli ${ }^{\text {am }}$, F. Cindolo ${ }^{\text {h}}$, I. Clare ${ }^{\mathrm{m}}$, R. Clare ${ }^{\text {ak }}$, G. Coignet ${ }^{\mathrm{d}}$, N. Colino ${ }^{\mathrm{x}}$, S. Costantini ${ }^{\text {al }}$, B. de la Cruz ${ }^{\mathrm{x}}$, S. Cucciarelli ${ }^{\text {af }}$, J.A. van Dalen ${ }^{\text {ad }}$, R. de Asmundis ${ }^{\mathrm{ab}}$, P. Déglon ${ }^{\mathrm{t}}$, J. Debreczeni ${ }^{1}$, A. Degré ${ }^{\mathrm{d}}$, K. Dehmelt ${ }^{\mathrm{y}}$, K. Deiters ${ }^{\mathrm{ar}}$, D. della Volpe ${ }^{\text {ab }}$, E. Delmeire ${ }^{t}$, P. Denes ${ }^{\text {aj }}$, F. DeNotaristefani ${ }^{\text {al }}$, A. De Salvo ${ }^{\text {at }}$, M. Diemoz ${ }^{\mathrm{al}}$, M. Dierckxsens ${ }^{\mathrm{b}}$, C. Dionisi ${ }^{\mathrm{al}}$, M. Dittmar ${ }^{\mathrm{at}, \mathrm{r}}$, A. Doria ${ }^{\mathrm{ab}}$, M.T. Dova ${ }^{\mathrm{j}, 5}$, D. Duchesneau ${ }^{d}$, M. Duda a , B. Echenard ${ }^{t}$, A. Eline ${ }^{r}$, A. El Hage ${ }^{\text {a }}$, H. El Mamouni ${ }^{\text {w, }}$ A. Engler ${ }^{\text {ah }}$, F.J. Eppling ${ }^{\mathrm{m}}$, P. Extermann ${ }^{\mathrm{t}}$, M.A. Falagan ${ }^{\mathrm{x}}$, S. Falciano ${ }^{\text {al }}$, A. Favara ${ }^{\text {ae }}$, J. Fay ${ }^{\text {w }}$, O. Fedin ${ }^{\text {ag }}$, M. Felcini ${ }^{\text {at }}$, T. Ferguson ${ }^{\text {ah }}$, H. Fesefeldt ${ }^{\text {a }}$, E. Fiandrini ${ }^{\text {af }}$, J.H. Field ${ }^{\mathrm{t}}$, F. Filthaut ${ }^{\text {ad }}$, P.H. Fisher ${ }^{\mathrm{m}}$, W. Fisher ${ }^{\text {aj }}$, I. Fisk ${ }^{\text {an }}$, G. Forconi ${ }^{\mathrm{m}}$, K. Freudenreich ${ }^{\text {at }}$, C. Furetta ${ }^{\mathrm{z}}$, Yu. Galaktionov ${ }^{\mathrm{a} a, \mathrm{~m}}$, S.N. Ganguli ${ }^{\mathrm{i}}$, P. Garcia-Abia ${ }^{\mathrm{e}, \mathrm{r}}$, M. Gataullin ${ }^{\text {ae }}$, S. Gentile al, S. Giagu ${ }^{\text {al }}$, Z.F. Gong ${ }^{\text {u }}$, G. Grenier ${ }^{\text {w }}$, O. Grimm ${ }^{\text {at }}$, M.W. Gruenewald ${ }^{p}$, M. Guida ${ }^{\text {am }}$, R. van Gulik ${ }^{\text {b }}$, V.K. Gupta ${ }^{\text {aj }}$, A. Gurtu ${ }^{\text {i }}$, L.J. Gutay ${ }^{\text {aq }}$, D. Haas ${ }^{\mathrm{e}}$, R.Sh. Hakobyan ${ }^{\text {ad }}$, D. Hatzifotiadou ${ }^{\text {h }}$, T. Hebbeker ${ }^{\mathrm{a}}$, A. Hervé ${ }^{\mathrm{r}}$, J. Hirschfelder ${ }^{\text {ah }}$, H. Hofer ${ }^{\text {at }}$, M. Hohlmann ${ }^{\mathrm{y}}$, G. Holzner ${ }^{\text {at }}$, S.R. Hou ${ }^{\text {av }}, \mathrm{Y}_{\mathrm{Hu}}{ }^{\text {ad }}$, B.N. Jin ${ }^{\mathrm{g}}$, L.W. Jones ${ }^{\mathrm{c}}$, P. de Jong ${ }^{\mathrm{b}}$, I. Josa-Mutuberría ${ }^{\mathrm{x}}$, D. Käfer ${ }^{\mathrm{a}}$, M. Kaur ${ }^{\mathrm{n}}$, M.N. Kienzle-Focacci ${ }^{\mathrm{t}}$, J.K. Kim ${ }^{\text {ap }}$, J. Kirkby ${ }^{\mathrm{r}}$, W. Kittel ${ }^{\text {ad }}$, A. Klimentov ${ }^{\mathrm{m} \text {,aa }}$, A.C. König ad, M. Kopal ${ }^{\text {aq }}$, V. Koutsenko ${ }^{\text {m,aa }}$, M. Kräber ${ }^{\text {at }}$, R.W. Kraemer ${ }^{\text {ah }}$, A. Krüger ${ }^{\text {as }}$, A. Kunin ${ }^{\mathrm{m}}$, P. Ladron de Guevara ${ }^{\mathrm{x}}$, I. Laktineh ${ }^{\mathrm{w}}$, G. Landi ${ }^{\mathrm{q}}$, M. Lebeau ${ }^{\mathrm{r}}$, 
A. Lebedev ${ }^{\mathrm{m}}$, P. Lebrun ${ }^{\mathrm{w}}$, P. Lecomte ${ }^{\text {at }}$, P. Lecoq $^{\mathrm{r}}$, P. Le Coultre ${ }^{\text {at }}$, J.M. Le Goff ${ }^{\mathrm{r}}$,

R. Leiste ${ }^{\text {as }}$, M. Levtchenko ${ }^{z}$, P. Levtchenko ${ }^{\text {ag }}$, C. Li ${ }^{u}$, S. Likhoded ${ }^{\text {as }}$, C.H. Lin ${ }^{\text {av }}$, W.T. Lin ${ }^{\text {av }}$, F.L. Linde ${ }^{b}$, L. Lista ${ }^{\text {ab }}$, Z.A. Liu ${ }^{g}$, W. Lohmann ${ }^{\text {as }}$, E. Longo ${ }^{\text {al }}$, Y.S. Lu ${ }^{g}$, C. Luci ${ }^{\text {al }}$, L. Luminari ${ }^{\text {al }}$, W. Lustermann ${ }^{\text {at }}$, W.G. Ma ${ }^{\text {u }}$, L. Malgeri ${ }^{t}$, A. Malinin ${ }^{\text {aa }}$, C. Maña ${ }^{\mathrm{x}}$, D. Mangeol ${ }^{\text {ad }}$, J. Mans ${ }^{\text {aj }}$, J.P. Martin ${ }^{\text {w }}$, F. Marzano ${ }^{\text {al }}$, K. Mazumdar ${ }^{\mathrm{i}}$, R.R. McNeil ${ }^{\mathrm{f}}$, S. Mele ${ }^{\mathrm{r}, \mathrm{ab}}$, L. Merola ${ }^{\text {ab }}$, M. Meschini ${ }^{\mathrm{q}}$, W.J. Metzger ${ }^{\text {ad }}$, A. Mihul ${ }^{\mathrm{k}}$, H. Milcent ${ }^{\mathrm{r}}$, G. Mirabelli ${ }^{\text {al }}$, J. Mnich ${ }^{\mathrm{a}}$, G.B. Mohanty ${ }^{\mathrm{i}}$, G.S. Muanza ${ }^{\mathrm{w}}$, A.J.M. Muijs ${ }^{\mathrm{b}}$, B. Musicar ${ }^{\text {an }}$, M. Musy al ${ }^{\text {al }}$ S. Nagy ${ }^{\circ}$, S. Natale ${ }^{t}$, M. Napolitano ${ }^{\text {ab }}$, F. Nessi-Tedaldi ${ }^{\text {at }}$, H. Newman ${ }^{\text {ae }}$, A. Nisati ${ }^{\text {al }}$, H. Nowak ${ }^{\text {as }}$, R. Ofierzynski ${ }^{\text {at }}$, G. Organtini ${ }^{\text {al }}$, C. Palomares ${ }^{\mathrm{r}}$, P. Paolucci ${ }^{\text {ab }}$, R. Paramatti ${ }^{\text {al }}$, G. Passaleva ${ }^{\text {q }}$, S. Patricelli ${ }^{\text {ab }}$, T. Paul ${ }^{\mathrm{j}}$, M. Pauluzzi $^{\text {af }}$, C. Paus ${ }^{\mathrm{m}}$, F. Pauss ${ }^{\text {at }}$, M. Pedace ${ }^{\text {al }}$, S. Pensotti ${ }^{\mathrm{z}}$, D. Perret-Gallix ${ }^{\mathrm{d}}$, B. Petersen ${ }^{\text {ad }}$, D. Piccolo ${ }^{\text {ab }}$, F. Pierella ${ }^{\text {h }}$, M. Pioppi ${ }^{\text {af }}$, P.A. Piroué ${ }^{\text {aj }}$, E. Pistolesi ${ }^{z}$, V. Plyaskin ${ }^{\text {aa }}$, M. Pohl ${ }^{\mathrm{t}}$, V. Pojidaev ${ }^{\mathrm{q}}$, J. Pothier ${ }^{\mathrm{r}}$, D.O. Prokofiev ${ }^{\mathrm{aq}}$, D. Prokofiev ${ }^{\mathrm{ag}}$, J. Quartieri ${ }^{\mathrm{am}}$, G. Rahal-Callot ${ }^{\text {at }}$, M.A. Rahaman ${ }^{\text {i, P. Raics }}{ }^{\circ}$, N. Raja ${ }^{\text {i }, ~ R . ~ R a m e l l i ~}{ }^{\text {at }}$, P.G. Rancoita ${ }^{\text {z }}$, R. Ranieri ${ }^{\mathrm{q}}, \mathrm{A}$. Raspereza ${ }^{\text {as }}, \mathrm{P}$. Razis ${ }^{\text {ac }}$, D. Ren ${ }^{\text {at }}, \mathrm{M}$. Rescigno ${ }^{\text {al }}, \mathrm{S}$. Reucroft ${ }^{\mathrm{j}}$, S. Riemann ${ }^{\text {as }}$, K. Riles ${ }^{c}$, B.P. Roe ${ }^{c}$, L. Romero $^{\mathrm{x}}$, A. Rosca ${ }^{\text {as }}$, S. Rosier-Lees ${ }^{\mathrm{d}}$, S. Roth ${ }^{\text {a }}$, C. Rosenbleck ${ }^{\text {a }}$, B. Roux ${ }^{\text {ad }}$, J.A. Rubio ${ }^{\text {r }}$, G. Ruggiero ${ }^{\mathrm{q}}$, H. Rykaczewski ${ }^{\text {at }}$, A. Sakharov ${ }^{\text {at }}$, S. Saremi ${ }^{\text {f }}$, S. Sarkar ${ }^{\text {al }}$, J. Salicio ${ }^{\text {r }}$, E. Sanchez ${ }^{\mathrm{x}}$, M.P. Sanders ${ }^{\text {ad }}$, C. Schäfer ${ }^{\mathrm{r}}$, V. Schegelsky ${ }^{\mathrm{ag}}$, H. Schopper ${ }^{\text {au }}$, D.J. Schotanus ${ }^{\mathrm{ad}}$, C. Sciacca ${ }^{\mathrm{ab}}$, L. Servoli af, S. Shevchenko ae, N. Shivarov ${ }^{\text {ao }}$, V. Shoutko ${ }^{\mathrm{m}}$, E. Shumilov ${ }^{\text {aa }}$, A. Shvorob ${ }^{\text {ae }}$, D. Son ${ }^{\text {ap }}$, C. Souga ${ }^{\text {w }}$, P. Spillantini ${ }^{\text {q }}$, M. Steuer ${ }^{\mathrm{m}}$, D.P. Stickland ${ }^{\text {aj }}$, B. Stoyanov ao, A. Straessner ${ }^{\text {r }}$ K. Sudhakar ${ }^{i}$, G. Sultanov ${ }^{\text {ao }}$, L.Z. Sun ${ }^{\text {u }}$, S. Sushkov ${ }^{\text {a }}$, H. Suter ${ }^{\text {at }}$, J.D. Swain ${ }^{\mathrm{j}}$, Z. Szillasi ${ }^{\mathrm{y}, 3}$, X.W. Tang ${ }^{\mathrm{g}}$, P. Tarjan ${ }^{\mathrm{o}}$, L. Tauscher ${ }^{\mathrm{e}}$, L. Taylor ${ }^{\mathrm{j}}$, B. Tellili ${ }^{\mathrm{w}}$, D. Teyssier ${ }^{\mathrm{w}}$, C. Timmermans ${ }^{\mathrm{ad}}$, Samuel C.C. Ting ${ }^{\mathrm{m}}$, S.M. Ting ${ }^{\mathrm{m}}$, S.C. Tonwar ${ }^{\mathrm{i}, \mathrm{r}}$, J. Tóth ${ }^{1}$, C. Tully ${ }^{\text {aj }}$, K.L. Tung ${ }^{\mathrm{g}}$, J. Ulbricht ${ }^{\text {at }}$, E. Valente ${ }^{\text {al }}$, R.T. Van de Walle ${ }^{\text {ad }}$, R. Vasquez ${ }^{\text {aq }}$, V. Veszpremi ${ }^{\text {y }}$, G. Vesztergombi ${ }^{1}$, I. Vetlitsky ${ }^{\text {aa }}$, D. Vicinanza ${ }^{\text {am }}$, G. Viertel ${ }^{\text {at }}$, S. Villa ${ }^{\text {ak }}$, M. Vivargent ${ }^{\mathrm{d}}, \mathrm{S}$. Vlachos ${ }^{\mathrm{e}}$, I. Vodopianov ${ }^{\text {ag }}$, H. Vogel ${ }^{\text {ah }}$, H. $_{\text {Vogt }}{ }^{\text {as }}$, I. Vorobiev ${ }^{\text {ah,aa }}$, A.A. Vorobyov ${ }^{\text {ag }}$, M. Wadhwa ${ }^{\mathrm{e}}$, X.L. Wang ${ }^{\mathrm{u}}$, Z.M. Wang ", M. Weber ${ }^{\text {a }}$, P. Wienemann ${ }^{\text {a }}$, H. Wilkens ${ }^{\text {ad }}$, S. Wynhoff ${ }^{\text {aj }}$, L. Xia ${ }^{\text {ae }}$, Z.Z. Xu ${ }^{\text {u }}$, J. Yamamoto ${ }^{\text {c }}$, B.Z. Yang ${ }^{\text {u }}$, C.G. Yang ${ }^{\mathrm{g}}$, H.J. Yang ${ }^{\mathrm{c}}$, M. Yang ${ }^{\mathrm{g}}$, S.C. Yeh ${ }^{\mathrm{aw}}$,

An. Zalite ${ }^{\mathrm{ag}}$, Yu. Zalite ${ }^{\mathrm{ag}}$, Z.P. Zhang ${ }^{\mathrm{u}}$, J. Zhao ${ }^{\text {u }}$, G.Y. Zhu ${ }^{\mathrm{g}}$, R.Y. Zhu ${ }^{\text {ae }}$, H.L. Zhuang ${ }^{\mathrm{g}}$, A. Zichichi ${ }^{\text {h,r,s }}$, B. Zimmermann ${ }^{\text {at }}$, M. Zöller ${ }^{\mathrm{a}}$.

\footnotetext{
${ }^{\text {a } I I I . ~ P h y s i k a l i s c h e s ~ I n s t i t u t, ~ R W T H, ~ D-52056 ~ A a c h e n, ~ G e r m a n y ~}{ }^{1}$

${ }^{\mathrm{b}}$ National Institute for High Energy Physics, NIKHEF, and University of Amsterdam, NL-1009 DB Amsterdam, The Netherlands c University of Michigan, Ann Arbor, MI 48109, USA

d Laboratoire d'Annecy-le-Vieux de Physique des Particules, LAPP,IN2P3-CNRS, BP 110, F-74941 Annecy-le-Vieux cedex, France

${ }^{\mathrm{e}}$ Institute of Physics, University of Basel, CH-4056 Basel, Switzerland

${ }^{\mathrm{f}}$ Louisiana State University, Baton Rouge, LA 70803, USA

$\mathrm{g}$ Institute of High Energy Physics, IHEP, 100039 Beijing, China 6

${ }^{\mathrm{h}}$ University of Bologna and INFN-Sezione di Bologna, I-40126 Bologna, Italy
} 


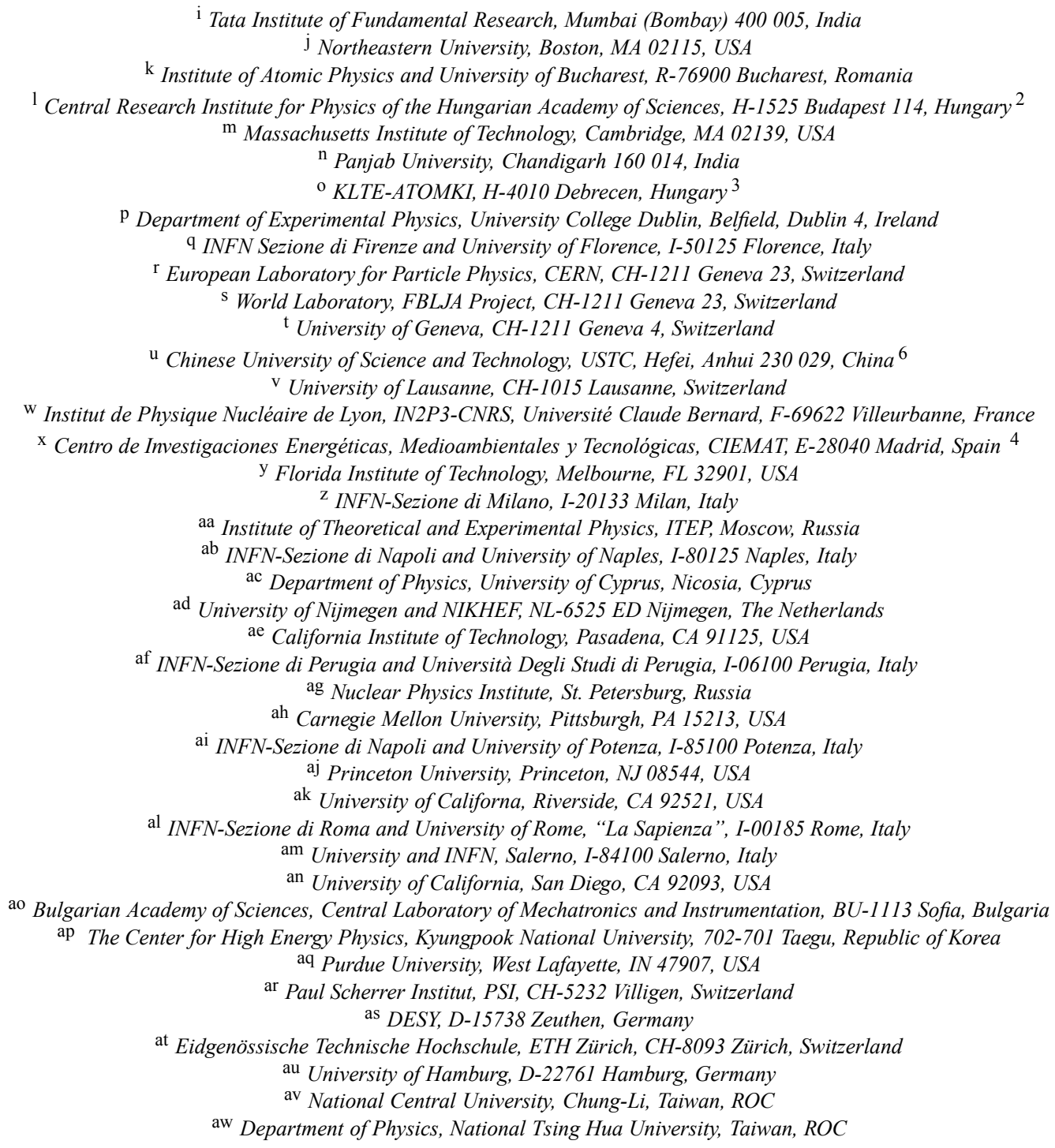

Received 25 October 2002; received in revised form 13 June 2003; accepted 27 June 2003

Editor: L. Rolandi

\begin{abstract}
A Higgs particle produced in association with a $\mathrm{Z}$ boson and decaying into weak boson pairs is searched for in $336.4 \mathrm{pb}^{-1} \mathrm{of}$ data collected by the L3 experiment at LEP at centre-of-mass energies from 200 to $209 \mathrm{GeV}$. Limits on the branching fraction of the Higgs boson decay into two weak bosons as a function of the Higgs mass are derived. These results are combined with the L3 search for a Higgs boson decaying to photon pairs. A Higgs produced with a Standard Model $\mathrm{e}^{+} \mathrm{e}^{-} \rightarrow \mathrm{Zh}$ cross section and decaying only into electroweak boson pairs is excluded at 95\% CL for a mass below $107 \mathrm{GeV}$.
\end{abstract} ○ 2003 Published by Elsevier B.V. 


\section{Introduction}

In the Standard Model, the Higgs mechanism generates the masses of elementary particles and stabilises the high energy behaviour of the electroweak interactions. However, the Standard Model does not predict the mass of the Higgs boson. Searches to date have focused on $b$ quark decays of the Higgs, but the model predicts an increased branching fraction to massive vector boson pairs for a heavier Higgs. In some extensions of the Standard Model which predict multiple Higgs bosons, the lightest Higgs boson couples primarily to bosons, not fermions [1]. Results excluding these "fermiophobic" models were reported for Higgs decays into two photons, but for Higgs masses above $90 \mathrm{GeV}$, the decay into massive vector boson pairs dominates [2].

Several possible models predict the presence of a fermiophobic Higgs. As a point of reference, a simple extension of the Standard Model is chosen where the production cross section for $\mathrm{e}^{+} \mathrm{e}^{-} \rightarrow \mathrm{Zh}$, the Higgsstrahlung process, is kept at the Standard Model value. All direct decays into fermions are removed, and the resulting branching ratios favour decays into two photons for Higgs bosons with masses below $90 \mathrm{GeV}$, while massive vector boson pairs become important above $90 \mathrm{GeV}$. This set of assumptions is called the "benchmark fermiophobic scenario". The branching ratios to $\gamma \gamma, \mathrm{W}^{+} \mathrm{W}^{-}$, and $\mathrm{ZZ}$ predicted in this scenario are plotted in Fig. 1.

This Letter presents the search for a Higgs boson produced in association with a $\mathrm{Z}$ boson through the process $\mathrm{e}^{+} \mathrm{e}^{-} \rightarrow \mathrm{Zh}$, followed by the decay of the Higgs to either a pair of $\mathrm{W}$ or $\mathrm{Z}$ bosons. Throughout the analysis we assume that the ratio between the branching fraction $\mathrm{h} \rightarrow \mathrm{WW}^{*}$ and the branching

\footnotetext{
${ }^{1}$ Supported by the German Bundesministerium für Bildung, Wissenschaft, Forschung und Technologie.

2 Supported by the Hungarian OTKA fund under contract numbers T019181, F023259 and T037350.

3 Also supported by the Hungarian OTKA fund under contract number T026178.

${ }^{4}$ Supported also by the Comisión Interministerial de Ciencia y Tecnología.

5 Also supported by CONICET and Universidad Nacional de La Plata, CC 67, 1900 La Plata, Argentina.

6 Supported by the National Natural Science Foundation of China.
}

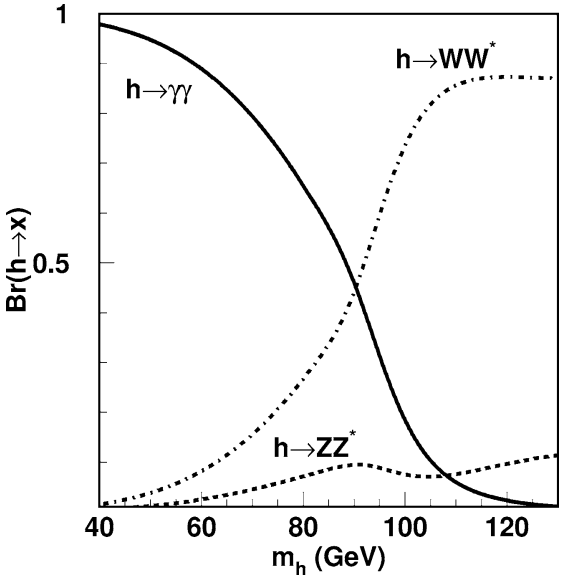

Fig. 1. Higgs branching fractions predicted in the benchmark fermiophobic scenario.

fraction $\mathrm{h} \rightarrow \mathrm{ZZ}^{*}$ is given by the Standard Model expectations. This assumption is valid for scenarios beyond the Standard Model satisfying the constraint $\rho \approx 1$ [3]. Consequently, the branching fraction $\mathrm{h} \rightarrow$ $\mathrm{WW}^{*}$ is expected to be the dominant weak boson decay for all Higgs masses. Accordingly, the analyses focus on $\mathrm{h} \rightarrow \mathrm{WW}^{*}$, but the efficiency of the analyses for a $\mathrm{h} \rightarrow \mathrm{ZZ}^{*}$ signal is considered where appropriate. Since the Higgsstrahlung mass reach of the LEP data is less than $160 \mathrm{GeV}$, at least one of the weak bosons produced from the Higgs decay must be off its mass shell. The analyses assume the presence of one vector boson with a mass within $\pm 10 \mathrm{GeV}$ of its nominal mass and one with a much smaller mass. This topology is expected to be present in approximately $70 \%$ of signal events.

\section{Data and Monte Carlo samples}

We analyse data collected with the L3 detector [4] at centre-of-mass energies $\sqrt{\mathrm{s}}=200-209 \mathrm{GeV}$, for a total integrated luminosity of $336.4 \mathrm{pb}^{-1}$. The data are grouped into six samples whose average centre-of-mass energies and corresponding integrated luminosities are listed in Table 1.

The Higgs signal cross section and fermiophobic branching ratios are calculated using the HZHA Monte Carlo generator [5]. For efficiency studies, Higgs events are generated using PYTHIA [6] for 
Table 1

Average centre-of-mass energies and corresponding integrated luminosities

\begin{tabular}{lcccccc}
\hline$\sqrt{s}(\mathrm{GeV})$ & 199.5 & 201.8 & 203.1 & 205.0 & 206.5 & 208.0 \\
\hline Luminosity $\left(\mathrm{pb}^{-1}\right)$ & 82.8 & 37.0 & 8.8 & 68.9 & 130.4 & 8.5 \\
\hline
\end{tabular}

Higgs masses between $80 \mathrm{GeV}$ and $120 \mathrm{GeV}$, with a mass step of $5 \mathrm{GeV}$ up to $100 \mathrm{GeV}$ and $1 \mathrm{GeV}$ thereafter. The $\mathrm{e}^{+} \mathrm{e}^{-} \rightarrow \mathrm{ZZ}, \mathrm{e}^{+} \mathrm{e}^{-} \rightarrow \mathrm{Ze}^{+} \mathrm{e}^{-}$, and $\mathrm{e}^{+} \mathrm{e}^{-} \rightarrow$ $\mathrm{q} \overline{\mathrm{q}}(\gamma)$ processes are simulated with the PYTHIA generator. The KK2f [7] generator is also used for the $\mathrm{e}^{+} \mathrm{e}^{-} \rightarrow \mathrm{q} \overline{\mathrm{q}}(\gamma)$ process. The KORALW [8] generator is used for the $\mathrm{e}^{+} \mathrm{e}^{-} \rightarrow \mathrm{W}^{+} \mathrm{W}^{-}$process except for the $\mathrm{e}^{+} \mathrm{e}^{-} \rightarrow \mathrm{e}^{-} \bar{\nu}_{\mathrm{e}} \mathrm{q} \overline{\mathrm{q}}^{\prime}$ final state, which is simulated using EXCALIBUR [9]. Hadron production in two-photon interactions is modelled using the PHOJET program [10].

The L3 detector response is simulated using the GEANT program [11], which takes into account the effects of energy loss, multiple scattering and showering in the detector. The GHEISHA package [12] is used to simulate hadronic interactions in the detector. Time dependent detector inefficiencies, as monitored during the data acquisition period, are also simulated.

\section{Analyses}

Of the possible final states from the $\mathrm{e}^{+} \mathrm{e}^{-} \rightarrow \mathrm{Zh} \rightarrow$ $\mathrm{ZWW}^{*} \rightarrow 6$ fermions process, this Letter describes the results of analyses for six of them, covering $93 \%$ of the total branching ratio. The six decay channels searched are: $\mathrm{Zh} \rightarrow$ qqqqqq, $\mathrm{Zh} \rightarrow$ qqqq $\ell \nu, \mathrm{Zh} \rightarrow$ $\mathrm{qq} \ell \nu \ell v, \mathrm{Zh} \rightarrow$ vvqqqq, $\mathrm{Zh} \rightarrow$ vvqq $\ell \nu$, and $\mathrm{Zh} \rightarrow$

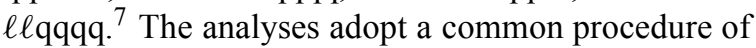
preselection, followed by a selection using a neural network approach and the production of a final discriminant variable.

The first step is to apply a set of preselection cuts to remove the background events most different from the signal. Important preselection cuts are made on visible energy and counts of tracks and clusters. The number of neutrinos in a channel determines the visible energy window, while the number of jets provides a basis for cuts on the number of charged tracks

\footnotetext{
7 This simplified notation, covering all possible combinations of flavour and charge, is adopted throughout this Letter.
}

and calorimeter clusters. For channels containing leptons, important lepton identification cuts are applied. Two-photon events and $\mathrm{e}^{+} \mathrm{e}^{-} \rightarrow \mathrm{q} \overline{\mathrm{q}}(\gamma)$ events containing one or more photons from initial state radiation are suppressed using cuts on the fraction of $\sqrt{s}$ deposited in detectors near the beam line and by cutting events where the missing momentum vector points near the beam pipe. These cuts also reduce the number of $\mathrm{e}^{+} \mathrm{e}^{-} \rightarrow$ evqq background events accepted by the analysis.

The background processes which are more similar to the signal are removed using one or more neural networks [13]. Where possible, preselected events are subjected to a constrained fit to the expected event topology before constructing the neural networks. Variables considered in the networks include the energies of the most and least energetic jets, the minimum angle between any two jets, the minimum angle between any jet and any lepton, and the reconstructed masses of any $\mathrm{Z}$ or $\mathrm{W}$ bosons identified in the event.

After applying cuts on the neural networks, the analyses produce final distributions of the selected signal, background, and data, generally using a discriminant combination of the neural network outputs and a reconstructed Higgs mass.

In the following sections we outline the selection procedures for the six channels. Complete details are available in Ref. [14].

\section{1. $\mathrm{Zh} \rightarrow \mathrm{qqqqqq}$}

The qqqqqq analysis searches for the case when the $\mathrm{W}$ and $\mathrm{Z}$ bosons decay into hadrons. The primary backgrounds to this channel are $\mathrm{ZZ} \rightarrow$ qqqq, $\mathrm{W}^{+} \mathrm{W}^{-} \rightarrow \mathrm{qqqq}$ and $\mathrm{e}^{+} \mathrm{e}^{-} \rightarrow \mathrm{q} \overline{\mathrm{q}}(\gamma)$ events. This sixjet signature is also produced by the $\mathrm{Zh} \rightarrow \mathrm{ZZZ}^{*} \rightarrow$ qqqqqq process, which represents $34 \%$ of the total $\mathrm{h} \rightarrow \mathrm{ZZ}^{*}$ signal. The efficiency for the $\mathrm{h} \rightarrow \mathrm{ZZ}^{*}$ signal is included in the analysis, effectively adding $15 \%$ to the expected rate relative to using only $\mathrm{h} \rightarrow \mathrm{WW}^{*}$.

Events with full energy and large hadronic content are selected. The analysis uses a fit of the event to 
a six-jet topology using the Durham algorithm [15]. Events with a poor match to the six-jet hypothesis are rejected.

After preselection, each six-jet event is subjected to a kinematic fit, which requires momentum and energy conservation. Following the fit, the pair of jets with invariant mass closest to $m_{Z}$ is chosen as the $\mathrm{Z}$ candidate. Of the remaining four jets, the dijet pair with invariant mass closest to $m_{\mathrm{W}}$ is assigned to be the $\mathrm{W}$ candidate and the remaining pair is identified as the $\mathrm{W}^{*}$.

The analysis uses three neural networks, one for each of the $\mathrm{e}^{+} \mathrm{e}^{-} \rightarrow \mathrm{W}^{+} \mathrm{W}^{-}, \mathrm{e}^{+} \mathrm{e}^{-} \rightarrow \mathrm{ZZ}$, and the $\mathrm{e}^{+} \mathrm{e}^{-} \rightarrow \mathrm{q} \overline{\mathrm{q}}(\gamma)$ backgrounds. All three neural networks use the same eleven inputs and have twenty hidden nodes and one output node. The input variables are chosen according to the specific features which differentiate signal from background:

- Typical QCD features in the background are: unequal repartition of the energy among the six jets, jets with low multiplicity, small invariant masses and small angles between closer jets. The following six input variables are therefore chosen: the energies of the most and least energetic jets, $E_{\text {jet }}^{\max }$ and $E_{\text {jet }}^{\min }$, the minimum number of tracks in a jet, $n_{\text {jet }}^{\min }$, the minimum angle between jets, $\theta_{j j}^{\min }$ and the values of the Durham algorithm parameter $\log y_{45}$ and $\log y_{56}$. The value $y_{m n}$ is the threshold for which the DURHAM algorithm reconstructs a $m$-jet event as a $n$-jet one.

- The event must be kinematically inconsistent with the production of only two on-shell boson pairs. This leads to the choice of the following five input variables: the chi-square of a kinematic fit to equalmass boson pair-production, $\chi_{\mathrm{WW}}^{2}$, the mass obtained in the fit, $m_{\text {eq }}^{\text {fit }}$, the masses of the $\mathrm{Z}$ and $\mathrm{W}$ candidates after kinematic fit to the signal hypothesis, $m_{Z}^{\text {fit }}$ and $m_{\mathrm{W}}^{\mathrm{fit}}$, and the angle between the decay planes of the $\mathrm{W}$ and $\mathrm{W}^{*}$ candidates, $\alpha_{\mathrm{WW}^{*}}$.

As an example of the input variables to the neural networks, Fig. 2(a) presents the distribution of $\theta_{j j}^{\min }$ for data, background and signal Monte Carlo. Table 2 gives the numbers of signal and background events expected and data observed after cuts on the neural network output. The final variable produced by the analysis is a discriminant combining the three network out- puts and the reconstructed Higgs mass after the kinematic fit and $\mathrm{Z}$ candidate assignment. Distributions of this final variable for data, background and signal are plotted in Fig. 3(a).

\section{2. $\mathrm{Zh} \rightarrow \mathrm{qqqq} \ell v$}

In the qqqqlv channel, the $\mathrm{Z}$ decays into hadrons, while one $\mathrm{W}$ decays into hadrons and the other decays into leptons. The different lepton flavours naturally define three different subchannels: qqqqe $v$, qqqq $\mu \nu$, and qqqq $\tau \nu$. Further, the difference between leptons coming from the $\mathrm{W}$ and from the $\mathrm{W}^{*}$ doubles the number of subchannels. In one set of signatures, the $\mathrm{W}$ decays into hadrons and the $\mathrm{W}^{*}$ decays into leptons, which means the lepton and neutrino energies are small, as is the missing energy of the event. In the other set, the $\mathrm{W}$ decays into leptons and the $\mathrm{W}^{*}$ decays into hadrons, yielding a high energy lepton and large missing energy. The major backgrounds are $\mathrm{W}$ pair production (especially $\left.\mathrm{e}^{+} \mathrm{e}^{-} \rightarrow \mathrm{W}^{+} \mathrm{W}^{-} \rightarrow \mathrm{qq} \tau \nu\right), \mathrm{Z}$ pair production, and the $\mathrm{e}^{+} \mathrm{e}^{-} \rightarrow \mathrm{q} \overline{\mathrm{q}}(\gamma)$ process.

Events are classified into a subchannel according to the most energetic lepton in the event. For the qqqqev and qqqq $\mu \nu$ cases, the subchannels are separated using the ratio of the lepton energy to the visible energy. In the subchannels where the lepton-neutrino pair comes from the on-shell $\mathrm{W}$, both the lepton and neutrino energies should be large. For the subchannels where the lepton and neutrino come from the $\mathrm{W}^{*}$, the converse is true. In the qqqq $\tau v$ channels, the initial lepton energy is difficult to reconstruct, so the subchannels are separated using only the visible energy. The candidate lepton in the event is required to meet minimum identification criteria to ensure isolated and well reconstructed leptons.

The analysis selects events with significant hadronic content and an appropriate amount of visible energy depending on the subchannel. A major source of background for this channel is the $\mathrm{W}^{+} \mathrm{W}^{-} \rightarrow$ $\mathrm{qq} \ell \nu$ process. To reduce it, an event is rejected if its hadronic mass is less than $90 \mathrm{GeV}$. After preselection, the major remaining background is $\mathrm{e}^{+} \mathrm{e}^{-} \rightarrow \mathrm{W}^{+} \mathrm{W}^{-}$, particularly $\mathrm{e}^{+} \mathrm{e}^{-} \rightarrow \mathrm{W}^{+} \mathrm{W}^{-} \rightarrow$ qqqq events with a lepton from hadron decays.

Two neural networks with ten input nodes are prepared for each lepton flavour to remove the $\mathrm{e}^{+} \mathrm{e}^{-} \rightarrow$ $\mathrm{W}^{+} \mathrm{W}^{-}$and $\mathrm{e}^{+} \mathrm{e}^{-} \rightarrow$ evqq backgrounds. Besides the 

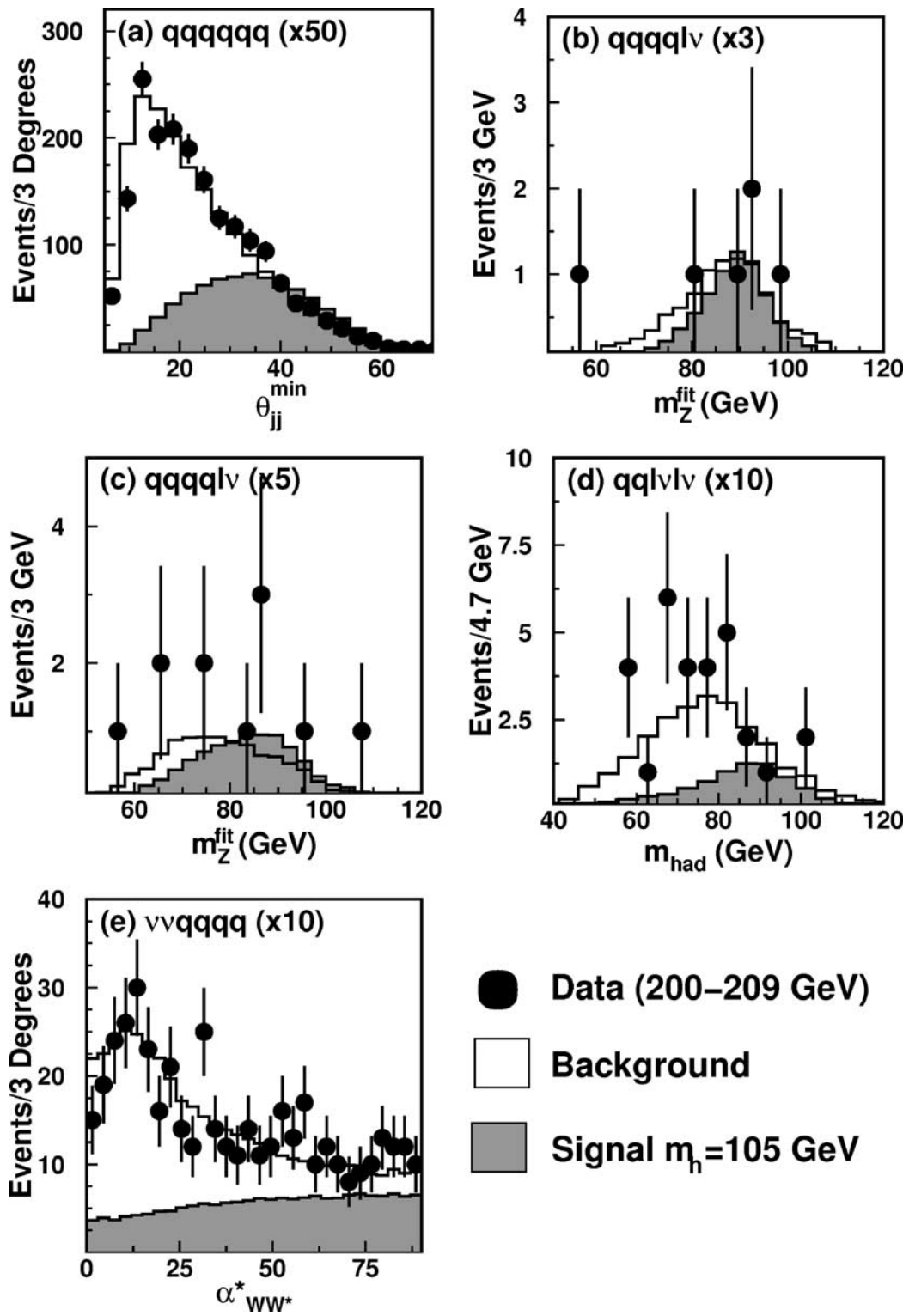

Data (200-209 GeV)

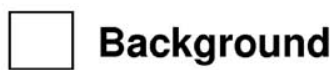

Signal $m_{h}=105 \mathrm{GeV}$

Fig. 2. Distributions of variables used in input to the neural networks for data, background and signal Monte Carlo at $m_{\mathrm{h}}=105 \mathrm{GeV}$. All centre-of-mass energies and subchannels are combined. (b) Refers to leptons originating from a W boson while (c) refers to leptons originating from a $\mathrm{W}^{*}$ boson. Signal Monte Carlo is magnified by the factor indicated in the figures.

Table 2

Number of events observed in data by the $\mathrm{Zh} \rightarrow$ qqqqqq analysis, compared with the Standard Model expectations. The Monte Carlo breakdown in different processes is given. Signal expectations are given for $m_{\mathrm{h}}=105 \mathrm{GeV}$ in the fermiophobic benchmark scenario

\begin{tabular}{lcccrrr}
\hline $\mathrm{Zh} \rightarrow$ qqqqqq & Data & Background & $\mathrm{W}^{+} \mathrm{W}^{-}$ & $\mathrm{ZZ}$ & $\mathrm{q} \overline{\mathrm{q}}(\gamma)$ & Signal \\
\hline Preselection & 1886 & 1870.1 & 1274.4 & 104.6 & 488.9 & 16.6 \\
Selection & 443 & 446.0 & 347.7 & 44.1 & 54.0 & 14.4 \\
\hline
\end{tabular}




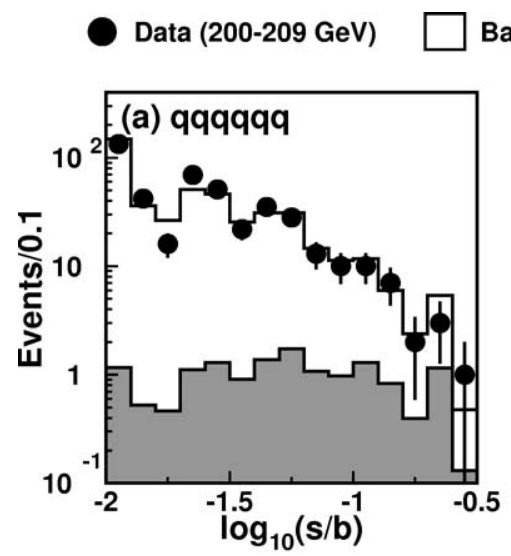

Background $\square$ Signal $m_{h}=105 \mathrm{GeV}$
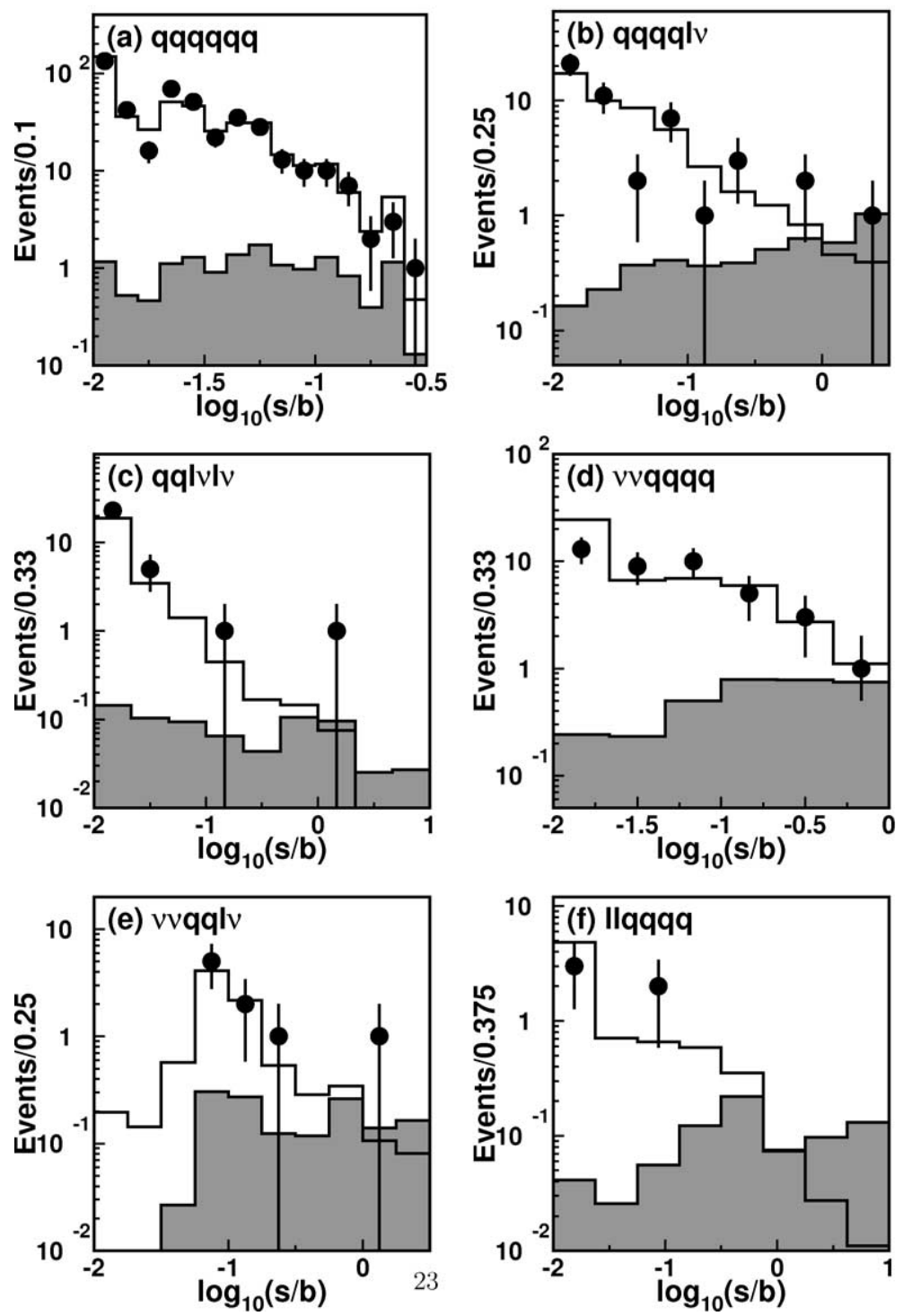

Fig. 3. Distributions of the final variable for data, background Monte Carlo and signal Monte Carlo at $m_{\mathrm{h}}=105 \mathrm{GeV}$. All centre-of-mass energies and subchannels are combined. The final variable is displayed in bins of $\log _{10}$ ( $\left.\frac{\text { signal }}{\text { background }}\right)$.

common input variables $E_{\text {jet }}^{\max }, E_{\text {jet }}^{\min }, \theta_{j j}^{\min }, \chi_{\mathrm{WW}}^{2}, m_{\mathrm{Z}}^{\mathrm{fit}}$ and $\log y_{34}$, the networks differ in the hypothesis used in associating the lepton-neutrino or quark-antiquark pairs to the $\mathrm{W}$ or the $\mathrm{W}^{(*)}$ bosons. For each network, four additional variables, constructed after a kinematic fit to the qqqq $\ell v$ topology, are used: the invariant masses of the lepton-neutrino and quark-antiquark candidate systems, $m_{\ell v}^{\mathrm{fit}}$ and $m_{\mathrm{qq}}^{\mathrm{fit}}$, as well as their respective momenta, $p_{\ell v}^{\mathrm{fit}}$ and $p_{\mathrm{qq}}^{\mathrm{fit}}$. These momenta are expected to be small for the signal hypothesis. Twenty hidden nodes and one output node are used for each network. The distributions in data, background and signal Monte Carlo of the variable $m_{\mathrm{Z}}^{\text {fit }}$ used in the neural networks are presented in Fig. 2(b) and (c) for 
Table 3

Number of events observed in data by the $\mathrm{Zh} \rightarrow \mathrm{qqqq} \ell \nu$ analysis, compared with the Standard Model expectations. The Monte Carlo breakdown in different processes is given. Signal expectations are given for $m_{\mathrm{h}}=105 \mathrm{GeV}$ in the fermiophobic benchmark scenario

\begin{tabular}{|c|c|c|c|c|c|c|c|}
\hline & & Data & Background & $\mathrm{w}^{+} \mathrm{W}^{-}$ & $\mathrm{ZZ}$ & $\mathrm{q} \overline{\mathrm{q}}(\gamma)$ & Signal \\
\hline \multirow[t]{2}{*}{$\mathrm{Zh} \rightarrow$ qqqqe $v$} & Preselection & 13 & 12.5 & 4.1 & 2.3 & 2.8 & 2.3 \\
\hline & Selection & 6 & 5.3 & 1.5 & 1.4 & 1.1 & 2.0 \\
\hline \multirow{2}{*}{$\mathrm{Zh} \rightarrow \mathrm{qqqq} \mu \nu$} & Preselection & 3 & 7.3 & 5.0 & 1.4 & 0.9 & 1.7 \\
\hline & Selection & 1 & 3.0 & 1.7 & 0.8 & 0.5 & 1.5 \\
\hline \multirow[t]{2}{*}{$\mathrm{Zh} \rightarrow \mathrm{qqqq} \tau \nu$} & Preselection & 251 & 202.4 & 137.5 & 13.9 & 43.4 & 1.6 \\
\hline & Selection & 41 & 41.2 & 24.4 & 2.8 & 8.6 & 1.3 \\
\hline
\end{tabular}

Table 4

Number of events observed in data by the $\mathrm{Zh} \rightarrow \mathrm{qq} \ell \nu \ell v$ analysis, compared with the Standard Model expectations. The Monte Carlo breakdown in different processes is given. Signal expectations are given for $m_{\mathrm{h}}=105 \mathrm{GeV}$ in the fermiophobic benchmark scenario

\begin{tabular}{llrrrrrr}
\hline & & Data & Background & $\mathrm{W}^{+} \mathrm{W}^{-}$ & $\mathrm{ZZ}$ & evqq & Signal \\
\hline $\mathrm{Zh} \rightarrow \mathrm{qq} \ell \nu \ell \nu$ & Preselection & 23 & 21.4 & 10.7 & 1.6 & 7.6 & 0.7 \\
& Selection & 2 & 1.4 & 0.5 & 0.5 & 0.2 & 0.5 \\
$\mathrm{Zh} \rightarrow \mathrm{qq} \tau \nu \ell \nu$ & Preselection & 96 & 91.7 & 57.3 & 6.4 & 20.8 & 0.6 \\
& Selection & 28 & 23.1 & 13.1 & 3.5 & 4.6 & 0.4 \\
\hline
\end{tabular}

the hypotheses of leptonic decays of the $\mathrm{W}$ and $\mathrm{W}^{(*)}$ bosons, respectively. The numbers of events expected and observed in this channel after cuts on the neural network outputs are listed in Table 3.

The mass of the Higgs boson is reconstructed from the recoil against the two jets which form the $\mathrm{Z}$ candidate. The final result of the analysis is a discriminant variable combining the output of the neural network and the reconstructed mass separately for each subchannel. Fig. 3(b) shows the distributions of the final variable with all six subchannels combined.

\section{3. $\mathrm{Zh} \rightarrow \mathrm{qq} \ell \nu \ell v$}

In the qq $\ell \nu \ell v$ channel, the $Z$ decays into hadrons while both $\mathrm{W}$ bosons decay into lepton-neutrino pairs. The analysis requires two identified leptons, one with more than $12 \mathrm{GeV}$ of energy and the second with energy greater than $10 \mathrm{GeV}$. The two neutrinos in the signal signature imply a visible energy window between $50 \%$ and $85 \%$ of $\sqrt{s}$. The analysis starts from events with hadronic content and rejects the twophoton and $\mathrm{e}^{+} \mathrm{e}^{-} \rightarrow \mathrm{q} \overline{\mathrm{q}}(\gamma)$ backgrounds as described above.

The dominant backgrounds after preselection are the $\mathrm{e}^{+} \mathrm{e}^{-} \rightarrow \mathrm{W}^{+} \mathrm{W}^{-}$and $\mathrm{e}^{+} \mathrm{e}^{-} \rightarrow$ evqq processes. They are suppressed by using a single neural network based on seven kinematic input variables, with sixteen hidden nodes and one output node. No constrained fit is performed for the $\mathrm{Zh} \rightarrow \mathrm{qq} \ell \nu \ell \nu$ hypothesis because the two neutrinos render the technique ineffective, but some of the input variables are similar to the ones employed in the previous sections: $\log \left(y_{23} / y_{34}\right)$, the hadronic invariant mass, $m_{\text {had }}$, which should be consistent with $m_{Z}$, the minimum angles between each of the leptons and the closest jet, $\theta_{l j}^{\mathrm{min}}$, expected to be small in the case of quark decays, the invariant masses of the lepton-neutrino system and of the remaining of the event after a kinematic fit to the $\mathrm{W}^{+} \mathrm{W}^{-} \rightarrow \mathrm{qq} \ell v$ hypothesis, $m_{\ell v}^{\text {fit }}$ and $m_{\text {rem }}^{\text {fit }}$, and the angle between leptons, $\theta_{l l}$, which should be high in the background case. Distributions of $m_{\text {had }}$ in data, background and signal Monte Carlo are presented in Fig. 2(d). The numbers of events expected and observed in this channel after a cut on the neural network output are listed in Table 4.

After selection, the events are divided into two groups: events where neither lepton is identified as a tau and events where at least one of the leptons is identified as a tau. Most background events fall into the second group. Final discriminant variables are constructed for these two subchannels separately, using the reconstructed Higgs mass and the neural network output. The Higgs mass is reconstructed by 
Table 5

Number of events observed in data by the $\mathrm{Zh} \rightarrow$ vvqqqq analysis, compared with the Standard Model expectations. The Monte Carlo breakdown in different processes is given. Signal expectations are given for $m_{\mathrm{h}}=105 \mathrm{GeV}$ in the fermiophobic benchmark scenario

\begin{tabular}{lcccrrrr}
\hline $\mathrm{Zh} \rightarrow$ vvqqqq & Data & Background & $\mathrm{W}^{+} \mathrm{W}^{-}$ & $\mathrm{ZZ}$ & $\mathrm{e} v \mathrm{qq}$ & $\mathrm{q} \overline{\mathrm{q}}(\gamma)$ & Signal \\
\hline Preselection & 451 & 439.7 & 263.2 & 25.3 & 73.6 & 75.9 & 4.0 \\
Selection & 41 & 47.9 & 41.5 & 4.9 & 4.4 & 6.9 & 3.3 \\
\hline
\end{tabular}

scaling the jet masses and energies by a common factor until the dijet mass is equal to $m_{Z}$, and then calculating the recoil mass against the dijet. Fig. 3(c) shows the distributions of the final variable for data, background and signal, with the two groups of events combined.

\section{4. $\mathrm{Zh} \rightarrow$ vvqqqq}

In the case where the $Z$ decays into two neutrinos and both W's decay into hadrons, the signature is four jets plus missing energy. The missing mass should be the $\mathrm{Z}$ mass and the visible mass the Higgs mass. As in the qqqqqq channel, the $\mathrm{Zh} \rightarrow \mathrm{ZZZ}^{*} \rightarrow$ vvqqqq events are included along with the $\mathrm{h} \rightarrow \mathrm{WW}^{*}$ signal. The selection accepts events where either the radiated Higgsstrahlung $\mathrm{Z}$ or the $\mathrm{Z}$ from the Higgs decays into neutrinos, but not the $Z^{*} \rightarrow \nu v$ case because of insufficient missing energy. The accepted signatures comprise $20 \%$ of the total $\mathrm{Zh} \rightarrow \mathrm{ZZZ}^{*}$ branching fraction, and their inclusion increases the expected signal rate by $15 \%$. Most background comes from $\mathrm{Z}$ and $\mathrm{W}$ pair production (especially $\mathrm{e}^{+} \mathrm{e}^{-} \rightarrow$ $\left.\mathrm{W}^{+} \mathrm{W}^{-} \rightarrow \mathrm{qq} \tau \nu\right)$, and the $\mathrm{e}^{+} \mathrm{e}^{-} \rightarrow \mathrm{q} \overline{\mathrm{q}}(\gamma)$ process.

The analysis starts from events with significant hadronic content as well as substantial missing energy, suppressing $\mathrm{e}^{+} \mathrm{e}^{-} \rightarrow \mathrm{q} \overline{\mathrm{q}}(\gamma)$ and two-photon processes. Events are constrained into a four-jet topology using the Durham algorithm. Events where any of the four jets contains no charged track or has an energy less than $6 \mathrm{GeV}$ are rejected.

The preselected events are subjected to a constrained kinematic fit assuming a balanced event with four jets and an invisible $Z$. The two jets with the invariant mass closest to $m_{\mathrm{W}}$ are considered as the W candidate dijet. Three separate neural networks are created and used to remove background processes. These three networks, based on ten input variables, twenty hidden nodes and one output node, are trained against $\mathrm{Z}$ and $\mathrm{W}$ pair production and the $\mathrm{e}^{+} \mathrm{e}^{-} \rightarrow$ $\mathrm{q} \overline{\mathrm{q}}(\gamma)$ process. The eight input variables used in the networks are: the recoil mass of the event, $m_{\text {rec }}$, which should be consistent with $m_{\mathrm{Z}}$ for signal events, $E_{\text {jet }}^{\max }$, $E_{\text {jet }}^{\min }, \theta_{j j}^{\min }, m_{\mathrm{W}}^{\mathrm{fit}}, \log y_{23}, \log y_{34}$ and $\alpha_{\mathrm{WW} *}^{*}$. The angle $\alpha_{\mathrm{WW}^{*}}^{*}$ corresponds to the angle $\alpha_{\mathrm{WW}^{*}}$ calculated in the rest frame of the hadronic system. Distributions in data, background and signal Monte Carlo of $\alpha_{\mathrm{WW}}^{*}$ are presented in Fig. 2(e). Table 5 gives the numbers of signal and background events expected and data observed after cuts on the neural network outputs.

The final variable is constructed by combining the outputs of the three neural networks with the reconstructed Higgs mass in a single discriminant. Fig. 3(d) shows the distributions of this final variable for data, background and signal.

\section{5. $\mathrm{Zh} \rightarrow v v \mathrm{qq} \ell v$}

In the $v v q q \ell v$ channel, the $\mathrm{Z}$ decays into neutrinos and the $\mathrm{WW}^{*}$ pair decays into two quarks, a lepton and a neutrino. The major backgrounds for this process depend on lepton flavour and include $\mathrm{W}$ and $\mathrm{Z}$ pair production, evqq and Zee final states, as well as hadronic two-photon processes. As in the qqqq $\ell v$ channel, the signal divides into six subchannels as a function of the lepton flavour and origin. The same variables and lepton identification requirements are used to separate the subchannels.

The small visible energy in this channel makes the suppression of two-photon processes particularly important. Stringent requirements against this background are applied and events with moderate hadronic content are selected.

After preselection, the most important background is $\mathrm{e}^{+} \mathrm{e}^{-} \rightarrow \mathrm{W}^{+} \mathrm{W}^{-} \rightarrow \mathrm{qq} \ell \nu$ (particularly qq $\tau \nu$ ). This background is rejected using several variables including the average reconstructed $\mathrm{W}$ pair mass, $m_{\mathrm{W}}^{\text {ave }}$. This variable is constructed by scaling the energies and masses of the two jets by a common factor so that the sum of the jet energy is $\sqrt{s} / 2$, as would be the case in a 
Table 6

Number of events observed in data by the $\mathrm{Zh} \rightarrow v v \mathrm{qq} \ell v$ analysis, compared with the Standard Model expectations. The Monte Carlo breakdown in different processes is given. Signal expectations are given for $m_{\mathrm{h}}=105 \mathrm{GeV}$ in the fermiophobic benchmark scenario

\begin{tabular}{llcccccc}
\hline & & Data & Background & $\mathrm{W}^{+} \mathrm{W}^{-}$ & $\mathrm{ZZ}$ & evqq & Signal \\
\hline $\mathrm{Zh} \rightarrow$ v vqq $\ell v$ & Preselection & 21 & 11.3 & 5.7 & 1.2 & 3.9 & 1.0 \\
& Selection & 2 & 1.7 & 1.0 & - & 0.7 & 0.8 \\
$\mathrm{Zh} \rightarrow$ v vqq $(\ell v)^{*}$ & Preselection & 39 & 44.4 & 34.4 & 3.6 & 5.4 & 0.6 \\
& Selection & 7 & 5.3 & 3.9 & 0.5 & 0.9 & 0.6 \\
\hline
\end{tabular}

Table 7

Number of events observed in data by the $\mathrm{Zh} \rightarrow \ell$ lqqqq analysis, compared with the Standard Model expectations. The Monte Carlo breakdown in different processes is given. Signal expectations are given for $m_{\mathrm{h}}=105 \mathrm{GeV}$ in the fermiophobic benchmark scenario

\begin{tabular}{llccccc}
\hline & & Data & Background & Zee & ZZ & Signal \\
\hline $\mathrm{Zh} \rightarrow$ eeqqqq & Preselection & 4 & 6.8 & 1.3 & 5.3 & 0.4 \\
& Selection & 2 & 3.0 & 0.7 & 2.3 & 0.4 \\
$\mathrm{Zh} \rightarrow \mu \mu \mathrm{qqqq}$ & Preselection & 13 & 13.7 & 7.2 & 5.5 & 0.4 \\
& Selection & 3 & 4.2 & 1.4 & 2.7 & 0.4 \\
\hline
\end{tabular}

$\mathrm{e}^{+} \mathrm{e}^{-} \rightarrow \mathrm{W}^{+} \mathrm{W}^{-} \rightarrow \mathrm{qq} \ell v$ event. Using these rescaled jets, the $\mathrm{W}$ mass is calculated as the average of the dijet invariant mass and the invariant mass of the lepton and the missing energy vector.

Each subchannel uses one neural network with eight input variables, twenty hidden nodes and one output node in order to remove the $\mathrm{W}^{+} \mathrm{W}^{-}$and evqq backgrounds. The neural network input variables include $m_{\mathrm{W}}^{\mathrm{ave}}$, the dijet invariant mass and the missing invariant mass. In addition, most of the remaining kinematic information in the event is used: the lepton energy, the hadronic energy, the minimum angle between the lepton and jets, the angle between the lepton and the dijet plane, and the angle between lepton and missing energy vector. The numbers of events predicted and observed after cuts on the neural network outputs are listed in Table 6 .

There are insufficient constraints to fully reconstruct the Higgs mass, so the visible mass is used as the final variable after a cut on the neural network. Fig. 3(e) shows the distributions of the final variable with all six subchannels combined.

\section{6. $\mathrm{Zh} \rightarrow \ell \ell \mathrm{qqqq}$}

In this channel, the $\mathrm{Z}$ decays into a pair of electrons or muons and hadronic $\mathrm{W}$ decays are considered. The event signature is two energetic leptons with the invariant mass of the $Z$, two energetic jets with an invariant mass near $m_{\mathrm{W}}$, and two lower energy jets. This signature is also produced by $6.5 \%$ of the $\mathrm{h} \rightarrow$ $\mathrm{ZZ}^{*}$ signal events.

The events are separated into two subchannels according to the lepton flavour. The analysis selects balanced energy events with significant hadronic content.

The preselected events are fit to a topology with two leptons and four jets and subjected to a kinematic fit requiring energy and momentum conservation. The eeqqqq subchannel uses a neural network which is trained against the $\mathrm{e}^{+} \mathrm{e}^{-} \rightarrow \mathrm{ZZ}$ and $\mathrm{e}^{+} \mathrm{e}^{-} \rightarrow$ Zee backgrounds. The $\mu \mu \mathrm{qqqq}$ subchannel uses two neural networks, one trained to discriminate against the $\mathrm{e}^{+} \mathrm{e}^{-} \rightarrow$ ZZ process and the other against $\mathrm{e}^{+} \mathrm{e}^{-} \rightarrow$ $\mathrm{W}^{+} \mathrm{W}^{-}$. The neural networks have six input nodes, twenty five hidden nodes and one output variable. The six input variables are: $E_{\mathrm{jet}}^{\max }, E_{\mathrm{jet}}^{\min }, \theta_{j j}^{\min }, \theta_{l j}^{\min }$, $\log Y_{34}$ and the invariant mass of the dilepton system, which should be consistent with $m_{Z}$. The number of background and signal events expected and data events observed after cuts on the neural network outputs are given in Table 7.

The final variable is a discriminant which combines the output of the $\mathrm{ZZ}$ rejection neural network with the Higgs mass reconstructed from the recoil against the lepton pair. Fig. 3(f) shows the final variable distributions with the two subchannels combined. 


\section{Results}

The sensitivity to a Higgs boson decaying into weak boson pairs is enhanced when all the previous analyses are combined. This combination considers each final variable as an independent Poisson counting experiment. A single statistical estimator from all channels is then built. This statistical estimator is the log-likelihood ratio [16]:

$-2 \ln Q=2 \sum_{i} s_{i}-2 \sum_{i} n_{i} \ln \left(1+\frac{s_{i}}{b_{i}}\right)$,

where $n_{i}$ is the number of data events observed in the $i$ th bin of the final variable, $s_{i}$ is the number of expected signal events and $b_{i}$ is the number of expected background events. The values of $n_{i}, b_{i}$ and $s_{i}$ depend on the Higgs mass hypothesis. The $-2 \ln Q$ values expected in the presence of background alone or both signal and background are determined by replacing $n_{i}$ by $b_{i}$ or $s_{i}+b_{i}$, respectively. The plot of the $-2 \ln Q$ as a function of the mass hypothesis is shown in Fig. 4. The dark band surrounding the data gives the size of the systematic uncertainties. The systematic dependence of the $-2 \ln Q$ distribution on detector quantities such as calorimeter energy scale and tracking efficiency is determined by shifting correlated variables in the analysis and propagating the effects to the signal and background hypothesis distributions. Other factors, such as signal and background Monte Carlo statistics and cross section uncertainties, are also included. Table 8 details the different sources of systematic and their contribution to the total systematic uncertainty on $-2 \ln Q$.

No significant excess indicating the presence of a Higgs boson decaying into $\mathrm{WW}^{*}$ or $\mathrm{ZZ}^{*}$ is ob- served in the data, which would manifest as a significant dip in the $-2 \ln Q$ distribution. Confidence levels for the absence of a signal are hence derived [16]. Fig. 5 shows the observed and expected 95\% confidence level (CL) limits as a function of the Higgs mass, assuming the Standard Model production cross section. In the assumption of $\mathrm{BR}\left(\mathrm{h} \rightarrow \mathrm{WW}^{*}\right)+$

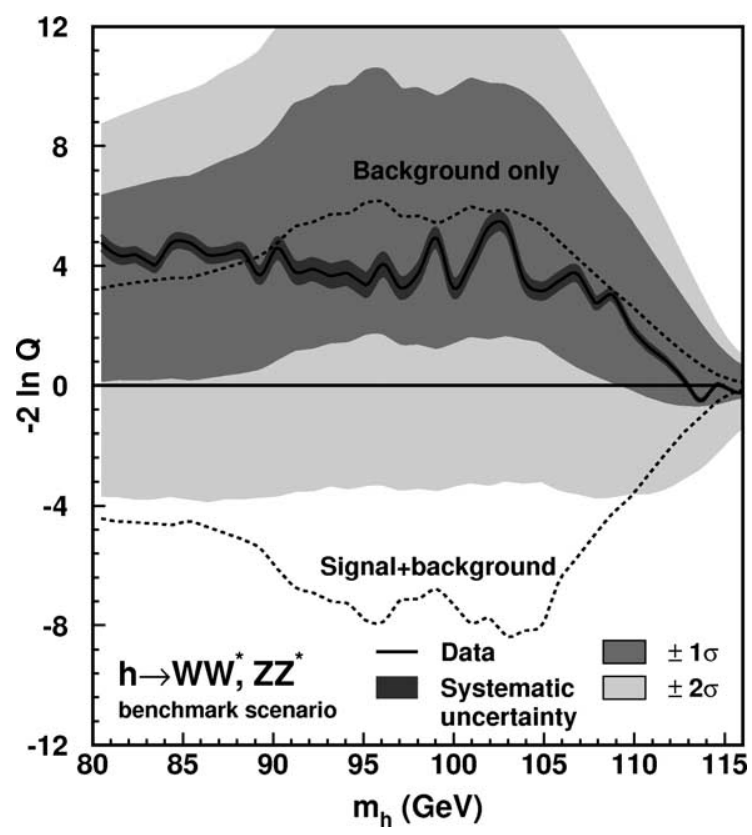

Fig. 4. Log-likelihood ratio plot for the combined $\mathrm{h} \rightarrow \mathrm{WW}^{*}, \mathrm{ZZ}^{*}$ search. The dashed lines represent the value of the expected background-only and signal + background distributions. The shaded regions around the background-only line indicate the $\pm 1 \sigma$ and $\pm 2 \sigma$ regions. The solid line indicates the observed values. The bands around the observed sensitivity represent the effects of systematic uncertainties.

Table 8

Systematic uncertainties on the $-2 \ln Q$ distributions for the different channels

\begin{tabular}{|c|c|c|c|c|c|c|}
\hline \multirow[t]{2}{*}{ Source of systematics } & \multicolumn{6}{|c|}{ Analysis $\mathrm{Zh} \rightarrow$} \\
\hline & qqqqqq (\%) & qqqq $\ell v(\%)$ & $\mathrm{qq} \ell \nu \ell v(\%)$ & vvqqqq $(\%)$ & $\nu v q q \ell v(\%)$ & $\ell \ell q q q q(\%)$ \\
\hline Tracking efficiency & 2.1 & 1.7 & 0.4 & 0.2 & 1.8 & 2.1 \\
\hline Energy scale & 1.3 & 2.9 & 2.0 & 6.0 & 5.3 & 2.2 \\
\hline Lepton identification & - & 3.8 & 2.8 & - & 4.4 & 1.8 \\
\hline Event shape & 1.2 & - & - & 2.6 & - & - \\
\hline Signal Monte Carlo statistics & 2.0 & 3.0 & 4.0 & 2.0 & 2.0 & 2.0 \\
\hline Background Monte Carlo statistics & 0.1 & 1.2 & 0.1 & 0.1 & 1.1 & 0.1 \\
\hline Background cross sections & 0.4 & 1.2 & 0.8 & 0.7 & 1.1 & 0.4 \\
\hline Total & 3.4 & 6.1 & 5.3 & 6.9 & 7.6 & 4.1 \\
\hline
\end{tabular}




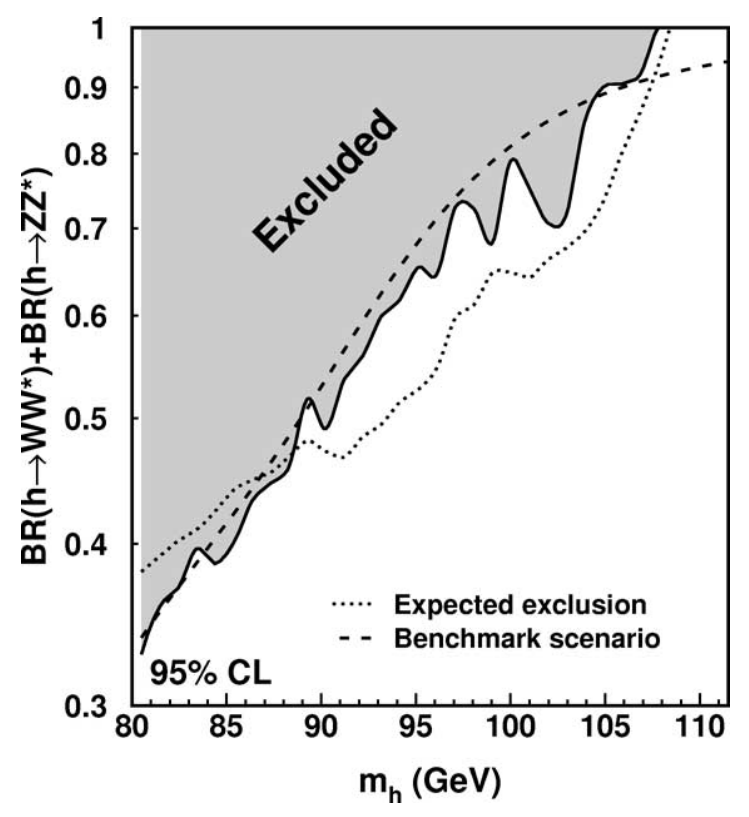

Fig. 5. Excluded values at $95 \% \mathrm{CL}$ of $\mathrm{BR}\left(\mathrm{h} \rightarrow \mathrm{WW}^{*}\right)+$ $\mathrm{BR}\left(\mathrm{h} \rightarrow \mathrm{ZZ}^{*}\right)$ as a function of the Higgs mass (solid line), in the assumption of the Standard Model production cross section. The expected 95\% CL limit (dashed line) and the fermiophobic benchmark prediction (dotted line) are also presented. The Standard Model prediction for $\mathrm{BR}\left(\mathrm{h} \rightarrow \mathrm{WW}^{*}\right)$ is $8 \%$ at $m_{\mathrm{h}}=115 \mathrm{GeV}$, falling below $1 \%$ for $m_{\mathrm{h}}<100 \mathrm{GeV}$.

$\mathrm{BR}\left(\mathrm{h} \rightarrow \mathrm{ZZ} \mathrm{Z}^{*}\right)=100 \%$, a Higgs boson with mass less than $108.1 \mathrm{GeV}$ is excluded at $95 \% \mathrm{CL}$. Assuming the value of $\mathrm{BR}\left(\mathrm{h} \rightarrow \mathrm{WW}^{*}\right)+\mathrm{BR}\left(\mathrm{h} \rightarrow \mathrm{ZZ}^{*}\right)$ of the benchmark fermiophobic scenario, calculated with the HDECAY program [17], the observed exclusion region is $83.7<m_{\mathrm{h}}<104.6 \mathrm{GeV}$ with a region between $88.9<m_{\mathrm{h}}<89.4 \mathrm{GeV}$ which can be excluded only at 93\% CL.

Model independent fermiophobic results can be derived by scanning the relative branching fractions of $\mathrm{h} \rightarrow \gamma \gamma$ and $\mathrm{h} \rightarrow \mathrm{WW}^{*}$. The branching fractions into boson pairs can be conveniently parameterised in the form

$$
\begin{aligned}
& \mathrm{BR}_{\text {bosons }}= \mathrm{BR}(\mathrm{h} \rightarrow \gamma \gamma)+\mathrm{BR}\left(\mathrm{h} \rightarrow \mathrm{WW}^{*}\right) \\
&+\mathrm{BRBR}\left(\mathrm{h} \rightarrow \mathrm{ZZ}^{*}\right), \\
& R_{\gamma \gamma}=\mathrm{BR}(\mathrm{h} \rightarrow \gamma \gamma) / \mathrm{BR}_{\text {bosons }} .
\end{aligned}
$$

$R_{\gamma \gamma}$ represents the fraction of fermiophobic decays into photon pairs, and ranges from zero to one, while $\mathrm{BR}_{\text {bosons }}$ represents the total Higgs branching

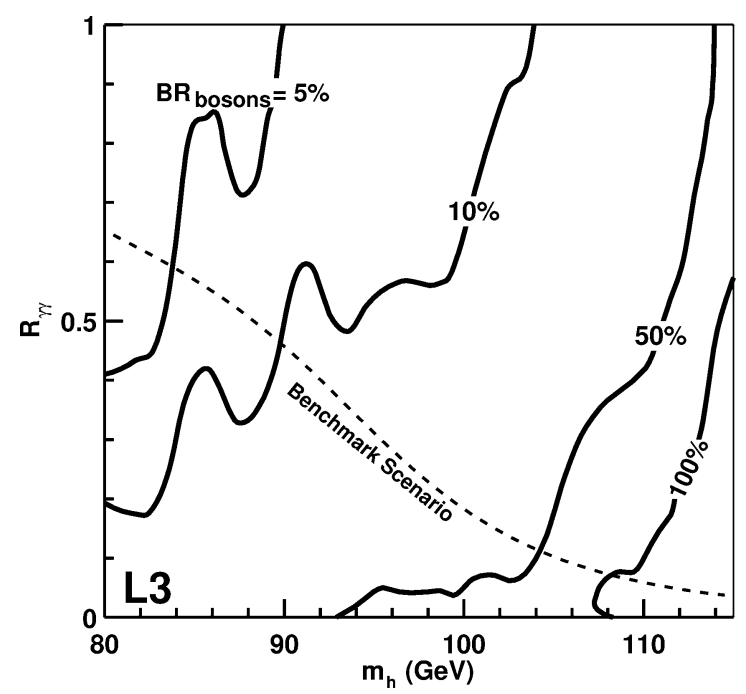

Fig. 6. The 95\% CL limit for $\mathrm{BR}_{\text {bosons }}$ as a function of $m_{\mathrm{h}}$ and $R_{\gamma \gamma}$. The solid lines indicate the borders between regions of exclusion. The crossing point between the $\mathrm{BR}_{\text {bosons }}=100 \%$ line and the dashed line provides the lower limit on the Higgs mass in the benchmark scenario: $m_{\mathrm{h}}>108.3 \mathrm{GeV}$. The minimum value of $m_{\mathrm{h}}$ on the $\mathrm{BR}_{\text {bosons }}=100 \%$ contour gives a lower mass limit for any model where the Higgs decays exclusively into electroweak boson pairs: $m_{\mathrm{h}}>107 \mathrm{GeV}$.

fraction to pairs of gauge bosons. The scan combines these $\mathrm{h} \rightarrow \mathrm{WW}^{*}$ results with the previously published $\mathrm{L} 3 \mathrm{~h} \rightarrow \gamma \gamma$ results [18], determining the $95 \% \mathrm{CL}$ exclusion for $\mathrm{BR}_{\text {bosons }}$ at each point in the $m_{\mathrm{h}}$ versus $R_{\gamma \gamma}$ plane. The full scan results are presented in Fig. 6 . In the benchmark scenario, the fermiophobic Higgs is limited at $95 \%$ CL to have $m_{\mathrm{h}}>108.3 \mathrm{GeV}$, compared to an expected limit of $m_{\mathrm{h}}>110.7 \mathrm{GeV}$. These results represent a significant extension of the $m_{\mathrm{h}}>$ 105.4 GeV limit obtained in the photonic channel [18]. These results also exclude at $95 \% \mathrm{CL}$ any model with $m_{\mathrm{h}}<107 \mathrm{GeV}$ for $\mathrm{BR}_{\text {bosons }}=100 \%$ and any value of $R_{\gamma \gamma}$, assuming the Standard Model production cross section.

\section{References}

[1] R. Santos, A. Barroso, Phys. Rev. D 56 (1997) 5366; L. Brücher, R. Santos, Eur. Phys. J. C 12 (2000) 87.

[2] M.A. Diaz, T.J. Weiler, hep-ph/9401259.

[3] J.F. Gunion, et al., The Higgs Hunter's Guide, AddisonWesley, 1990;

J.F. Gunion, R. Vega, J. Wudka, Phys. Rev. D 42 (1990) 1673; 
B. Grządkowski, J. Wudka, Phys. Lett. B 364 (1995) 49.

[4] L3 Collaboration, B. Adeva, et al., Nucl. Instrum. Methods A 289 (1990) 35;

J.A. Bakken, et al., Nucl. Instrum. Methods A 275 (1989) 81;

O. Adriani, et al., Nucl. Instrum. Methods A 302 (1991) 53;

B. Adeva, et al., Nucl. Instrum. Methods A 323 (1992) 109;

K. Deiters, et al., Nucl. Instrum. Methods A 323 (1992) 162;

M. Chemarin, et al., Nucl. Instrum. Methods A 349 (1994) 345;

M. Acciarri, et al., Nucl. Instrum. Methods A 351 (1994) 300;

G. Basti, et al., Nucl. Instrum. Methods A 374 (1996) 293;

A. Adam, et al., Nucl. Instrum. Methods A 383 (1996) 342.

[5] P. Janot, in: G. Altarelli, T. Sjöstrand, F. Zwirner (Eds.), Physics at LEP2, Report CERN 96-01, Vol. 2, 1996, p. 309.

[6] PYTHIA version 5.722 is used;

T. Sjöstrand, preprint CERN-TH/7112/93 (1993), revised 1995 ;

T. Sjöstrand, Comput. Phys. Commun. 82 (1994) 74.

[7] KK2F version 4.12 is used;

S. Jadach, B.F.L. Ward, Z. Wạs, Comput. Phys. Commun. 130 (2000) 260.

[8] KORALW version 1.33 is used;

M. Skrzypek, et al., Comput. Phys. Commun. 94 (1996) 216;

M. Skrzypek, et al., Phys. Lett. B 372 (1996) 289.

[9] EXCALIBUR version 1.11 is used;
F.A. Berends, R. Pittau, R. Kleiss, Comput. Phys. Commun. 85 (1995) 437.

[10] PHOJET version 1.05 is used;

R. Engel, Z. Phys. C 66 (1995) 203;

R. Engel, J. Ranft, S. Roesler, Phys. Rev. D 52 (1995) 1459.

[11] GEANT version 3.15 is used;

R. Brun, et al., preprint CERN DD/EE/84-1 (1984), revised 1987.

[12] H. Fesefeldt, RWTH Aachen report PITHA 85/02 (1985).

[13] SNNS: Stuttgart Neural Network Simulator, University of Stuttgart, http://www-ra.informatik.uni-tuebingen.de/SNNS.

[14] J. Mans, Search for a Higgs boson decaying to massive vector boson pairs at LEP, PhD thesis, Princeton/HEP/20022 (2002), hep-ex/0204029.

[15] S. Bethke, et al., Nucl. Phys. B 370 (1992) 310, and references therein.

[16] LEP Working Group for Higgs Boson Searches and ALEPH, DELPHI, L3 and OPAL Collaborations, preprint CERNEP/2000-55 (2000).

[17] A. Djouadi, J. Kalinowski, M. Spira, Comput. Phys. Commun. 108 (1998) 56.

[18] L3 Collaboration, P. Achard, et al., Phys. Lett. B 534 (2002) 28. 